\title{
Pull-out of Upper Thoracic Pedicle Screws Can Cause Spinal Canal Encroachment in Growing Rod Treatment
}

\author{
Senol Bekmez, MD, * Aykut Kocyigit, MD, $†$ Zeynep Deniz Olgun, MD, $\neq$ Mehmet Ayvaz, MD, $\S$ \\ Halil Gokhan Demirkiran, MD, $\S$ Ergun Karaagaoglu, MD,\| and Muharrem Yazici, MD $₫$
}

Background: Proximal foundation failure is a common complication of growing rod (GR) treatment for early-onset scoliosis. Spinal canal encroachment due to pull-out of pedicle screw used as proximal foundation has been anecdotally reported in GR patients. The aim of this study is to report the prevalence of spinal canal encroachment of pedicle screws in GR treatment and determine risk factors using a single-center cohort.

Methods: Inclusion criteria were: (1) GR for early-onset scoliosis and (2) pull-out of at least 1 proximal anchor pedicle screw. Patients were divided into 2 groups according to the presence of medial screw migration. Medial migration of the screw was confirmed by computed tomography. The extracted data included demographic, clinical, and radiographic information.

Results: A total of 21 patients (of 96) met inclusion criteria $(21.8 \%)$. None of the screws appeared malpositioned on early postoperative x-ray. Average follow-up until screw failure was 50.4 months (64 to $85 \mathrm{mo}$ ) and average number of lengthenings 8.1 (4 to 13). Computed tomography revealed canal encroachment in 11 patients (group 1), and no encroachment in 10 (group 2). There was no significant difference between groups for age, follow-up or number of lengthenings. At the time of screw pull-out, coronal plane deformity was increased compared with early postoperative $\mathrm{x}$-ray in all; however, this increase was significantly higher in group 1 (45.7 vs. 35 degrees, $P=0.002)$. Proximal junctional angle (PJA) was increased in both groups at the time of pull-out. While not statistically significant, PJA increased linearly in group 1 but spiked in group 2 at the time of pull-out. There was no neurological event preoperatively, intraoperatively or postoperatively. Failed screws were safely revised in either planned/ unplanned surgeries.

Conclusions: In patients with proximal anchor failure of GR, especially if there is increase of coronal deformity and/or PJA, possible spinal encroachment should be kept in mind.

Level of Evidence: Level IV - retrospective case series.

From the *Cankaya Hospital; Departments of †Orthopaedics and Traumatology; §Orthopaedics and Traumatology; \|Biostatistics; TOrthopaedics and Traumatology, Hacettepe University, Ankara, Turkey; and †Children’s Hospital of Pittsburgh of UPMC, Pittsburgh, PA.

No funds were received in support of this work

No relevant financial activities outside the submitted work.

The authors declare no conflicts of interest.

Reprints: Muharrem Yazici, MD, Department of Orthopaedics and Traumatology, Hacettepe University, Ankara 06100, Turkey. E-mail: mimyazici@gmail.com.

Copyright (C) 2018 Wolters Kluwer Health, Inc. All rights reserved.

DOI: $10.1097 /$ BPO.0000000000001196
Key Words: early-onset scoliosis, growing rods, growing rods complication, proximal anchor failure, spinal canal encroachment

( P Pediatr Orthop 2018;38:e399-e403)

$\mathrm{D}$ uring the past 2 decades, the growing rod (GR) technique has evolved as the fusionless procedure of choice to treat progressive early-onset scoliosis (EOS). Despite recent improvements in the technique, GR procedure is still prone to high complication rates, ranging from $29 \%$ to $58 \%$ in the literature. ${ }^{1-3}$ Proximal foundation dislodgement is one of the most frequent causes of implant-related complications. ${ }^{4,5}$

During the last decade, there has been a shift toward pedicle screws as proximal anchors to achieve a more secure fixation. Pedicle screws have been shown to be safe and reliable in adults as well as young children, ${ }^{6}$ and not to interfere with the growth of the vertebral body in the latter. ${ }^{7,8}$ Biomechanical studies have revealed that in the immature spine, pedicle screws constructs are the strongest in axial pull-out tests as compared with hooks or screwhook hybrids. ${ }^{9-11}$ Clinical studies also reported that pedicle screws lead to fewer implant-related complications compared with hooks in GR constructs. ${ }^{12}$ In contrast, spinal canal encroachment due to pull-out of pedicle screws used as proximal foundation has been anecdotally reported in GR patients. ${ }^{13,14}$ The aim of this study is to report the prevalence of this phenomenon and determine risk factors using a single-center cohort.

\section{METHODS}

All EOS patients undergoing treatment at our institution are included in a prospectively maintained database which includes demographic information as well as radiographic and surgical data. This database was accessed to find patients meeting the following inclusion criteria: (1) undergoing dual GR treatment for EOS, (2) containing at least 1 vertebra instrumented by bilateral pedicle screws in the proximal foundation, (3) undergoing revision surgery because of pull-out of an upper thoracic pedicle screw.

Proximal thoracic pedicle screws were placed with the freehand method as described by Kim et $\mathrm{al}^{15}$ and their position verified with fluoroscopy. Our proximal foundations include both pedicles of 2 thoracic vertebrae as appropriate for curve morphology. We try to utilize all pedicle screw constructs whenever possible; in the case of 
technical inability to place screws, we routinely utilize transverse process hooks at the same level. Supralaminar hooks were choice of implant at the time of revision if pedicle screw is failed.

At our facility, intraoperative neurological monitoring is routinely performed during index operations and procedures where new implant insertion is anticipated, but not for routine lengthening procedures. Screw pull-out was diagnosed with a plain lateral radiograph. When pull-out or loosening of a proximal screw was noted on plain radiographs, subsequent computed tomography (CT) was also performed to confirm orientation of the pedicle screw. Patients who were determined to have pull-out of the proximal pedicle screws were divided into 2 groups according to the presence of medial screw migration/canal encroachment.

The data extracted from our EOS database included demographic and clinical information, etiology, radiologic analysis, neurological status, surgical details of index and revision procedures, and the final status of the patient before the writing of this report. Patients' radiographic studies were reviewed, the magnitude of deformity, sagittal parameters, proximal junctional angle (PJA), and level of the failed screw noted. For this study, PJA was measured from the superior endplate of one vertebral level above the upper-instrumented vertebra to the inferior endplate of the upper-instrumented vertebra.

In statistical analysis, Mann-Whitney $U$ test was used for the comparison of numerical variables between 2 groups. For categorical variables, either Pearson $\chi^{2}$ of Fisher exact test was performed.

\section{RESULTS}

A total of 21 of 96 patients $(21.8 \%)$ met inclusion criteria. Average age at index surgery was 5.5 years (range, 3 to 8 y). Curve etiologies were idiopathic (9), congenital (7), syndromic (3), and neuromuscular (2).

None of the screws appeared malpositioned on early postoperative radigoraphs. Average follow-up was
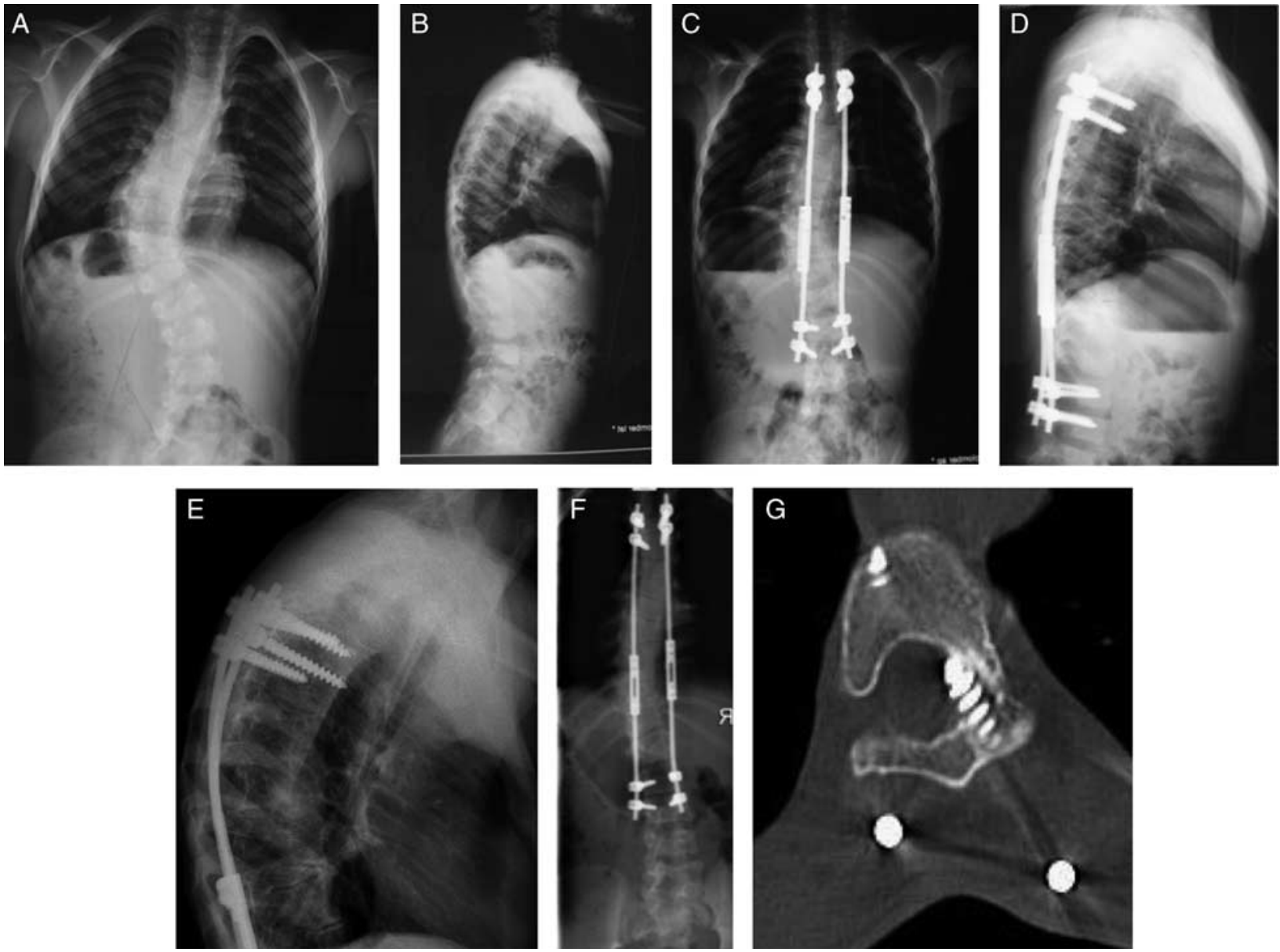

FIGURE 1. Anteroposterior (AP) (A) and lateral (B) radiographs of a 7-year-old male with idiopathic early-onset scoliosis. As postoperative $A P(C)$ and lateral (D) radiographs demonstrate, dual growing rod treatment was performed using pedicle screws as proximal and distal foundations. After 13 sequential lengthening procedures, pull-out of the left T5 pedicle screw was detected in the lateral radiograph (E) while AP radiographs appeared unremarkable (F). Axial computed tomographic scan (G) confirmed that there was canal encroachment by this pedicle screw. 

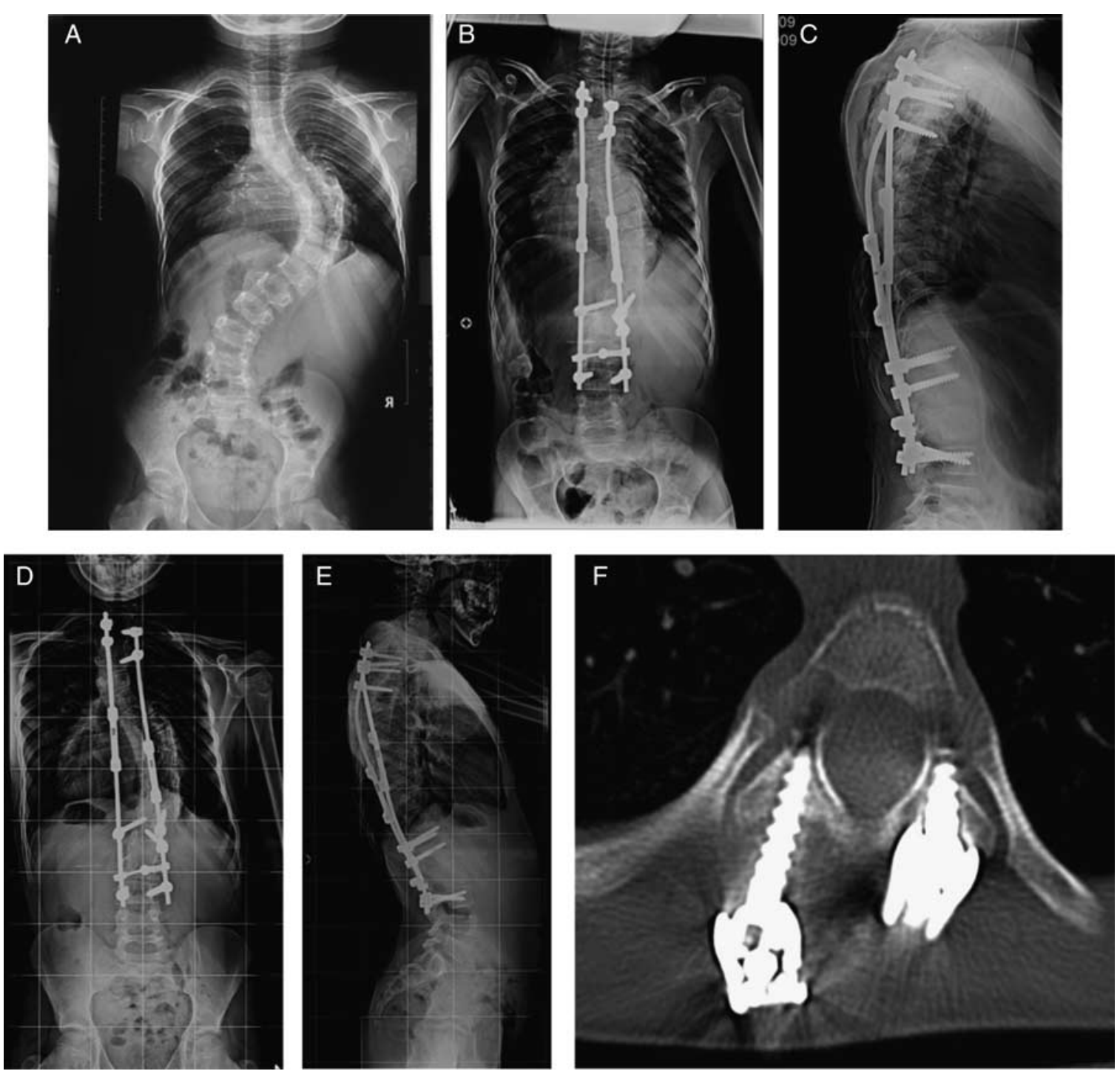

FIGURE 2. Anteroposterior (AP) (A) and lateral (B) radiographs of a 5-year-old male with idiopathic early-onset scoliosis treated with dual growing rod (C). After 5 sequential lenghtenings, AP radiographs appeared unremarkable (D) but pull-out of the rightsided T2 pedicle screw was detected in the lateral radiograph (E). Axial computed tomographic scan (F) revealed that the screw had not migrated into the spinal canal.

50.4 months (range, 64 to $85 \mathrm{mo}$ ) and patients had on average 8.1 lengthening procedures (range, 4 to 13 ) until proximal anchor pull-out. The levels of the failed screws were T2 (9), T3 (7), T4 (3), and T5 (2), respectively. CT

TABLE 1. Comparison of the Demographic and Operative Characteristics Between Groups

\begin{tabular}{lccl}
\hline Parameters & Group 1 & Group 2 & $\boldsymbol{P}$ \\
\hline Age (y) & 5.7 & 5.3 & 0.557 \\
Etiology (idiopathic/nonidiopathic) & $5 / 6$ & $4 / 6$ & 0.575 \\
Follow-up (mo) & 58.4 & 41.5 & 0.085 \\
No. screws in the proximal anchor & 3.8 & 3.7 & 0.45 \\
Level of failed screw (T3 and above/T4 & $8 / 3$ & $8 / 2$ & 0.55 \\
$\quad$ and below) & & & \\
Transverse connector (+/-) & $3 / 8$ & $3 / 7$ & 0.63 \\
No. lengthenings & 8.5 & 7.6 & 0.468 \\
\hline
\end{tabular}

revealed canal encroachment in 11 patients (group 1) (Fig. 1), and no encroachment in 10 (group 2) (Fig. 2). Two of 11 patients in group 1 and 3 of 10 in group 2 had a hybrid screw-transverse process hook construct for the proximal foundation, while the remaining patients in both groups had all-pedicle screw constructs.

There was no significant difference between groups for curve etiology, age at index surgery, follow-up time, number of pedicle screws at the proximal anchor, level of failed screw, presence of transverse connector or number of lengthening procedures (Table 1). Radiographic analysis of the groups is also summarized in the Table 2. At the time of screw pull-out, coronal plane deformity compared with early postoperative radiographs was increased in all; however, this increase was significantly higher in group 1 (45.7 vs. 35 degrees, $P=0.002$ ). PJA was 
TABLE 2. Comparison of the Radiographic Parameters Between Groups

\begin{tabular}{llcl}
\hline Parameters & Group 1 & Group 2 & $\boldsymbol{P}$ \\
\hline Magnitude of major curve (deg.) & & & \\
$\quad$ Preindex & 64 & 60.6 & 0.566 \\
Postindex & 35.2 & 30.3 & 0.260 \\
$\quad$ At screw pull-out & 45.7 & 35.6 & $0.002^{*}$ \\
T2-12 kyphosis (deg.) & & & \\
Preindex & 45 & 50.8 & 0.528 \\
Postindex & 34.4 & 31.4 & 0.389 \\
At pull-out & 44.2 & 46.7 & 0.551 \\
Kyphosis correction (\%) & 23.9 & 32.2 & 0.287 \\
PJA (deg.) & & & \\
Postindex & 5.9 & 4.9 & 0.331 \\
Last visit before pull-out & 9.2 & 7 & 0.189 \\
At screw pull-out & 14.5 & 12.3 & 0.449 \\
\hline
\end{tabular}

*Statistically significant difference.

PJA indicates proximal junctional angle.

markedly increased in both groups at the time of pull-out. While no statistical significance could reliably be demonstrated, PJA was noted to increase in a more gentle and linear manner in group 1 and demonstrate a sudden spike in group 2 at the time of pull-out $(P=0.269)$.

Operative notes and neurological monitoring records of all patients including index and revision surgery were also reviewed in detail. At index surgery, no abnormal signal was recorded in any of the patients during intraoperative neurological monitoring. During insertion of pedicle screws, no cerebrospinal fluid leakage or softness in the medial pedicle wall was noted. All screws were imaged with intraoperative fluoroscopy following insertion and none of them was seen to cross the midline or otherwise raise suspicion about a medial breach. There was no neurological deficit in any of the patients after index or lengthening procedures.

Once pedicle screw pull-out with or without spinal canal encroachment was diagnosed, revision surgery was performed expeditiously to remove the offending screw. At the time of revision, screw attachment to the rod was found to be secure in all patients. In 4 group 1 patients, we were able to replace the failed screw with a larger diameter but redirected screw at the same level. In 4 other group 1 patients, we were unable to place a new screw and a supralaminar hook was utilized at the same level. In 3 group 1 patients, the same level was unsuitable for anchor replacement and instrumentation was extended 1 level above, usually with screws. Again in 3 group 2 patients, failed screws were replaced by a larger diameter but redirected pedicle screw at the same level, in 5 patients by supralaminar hooks at the same level, and in 2 patients instrumentation was extended to 1 level above with pedicle screws. No abnormal signal was noted with neurological monitoring during revision surgery. Again, there was no postoperative neurological deficit in any of the patients after revision surgery.

\section{DISCUSSION}

Pull-out of proximal pedicle screw anchors is a potentially devastating complication in the course of growing rod treatment. The prevalence of and risk factors for this phenomenon have been addressed by previous studies. Watanabe et $\mathrm{al}^{4}$ reported that an increase of every 20 degrees in the proximal thoracic Cobb angle or thoracic kyphosis angle, and 6 or more lengthening procedures were independent risk factors for proximal anchor failure. The use of pedicle screws in the proximal foundation did not reduce these complication rates. In another study, Watanabe et $\mathrm{al}^{5}$ reported that, the prevalence of proximal foundation failure was $21.6 \%$. Proximal junctional kyphosis was a predisposing factor for proximal foundation dislodgement. They also described significant independent risk factors for proximal junctional kyphosis as proximal thoracic scoliosis of 40 degrees or more and main thoracic kyphosis of 60 degrees or more. Our study too demonstrates that pedicle screws at the proximal foundation of dual GRs constructs are prone to failure. The prevalence of screw pull-out was $21.8 \%$ in our series which is consistent with these previous studies.

Pedicle screws have been the gold standard in spinal instrumentation for the past 2 decades, including GR treatment. While minor medial breaches during freehand insertion of pedicle screws appears to be a frequent occurrence, ${ }^{16}$ actual clinical symptoms due to intraoperatively malpositioned pedicle screws are rare. Pedicle screws have been reported to be safe even in small children in several previous studies. ${ }^{6-8}$

Profound medial malposition of a pedicle screw at the proximal thoracic spine during long-term follow-up is a rare but potentially devastating complication. There is a scarcity of literature regarding this problem. Alanay et $\mathrm{al}^{13}$ reported a 6 -year-old girl with late spinal cord compression caused by $\mathrm{T} 2$ pedicle screw pull-out after posterior instrumented fusion. More recently, Skaggs et $\mathrm{al}^{14}$ reported patients with failure of thoracic pedicle screws 18 months after surgery, causing late spinal cord injury. In their report of three patients, 2 had undergone posterior instrumented fusion and 1 dual GR instrumentation. Their common finding was pull-out of pedicle screws from the first or second instrumented level when 5 or more levels below had been spanned without rigid fixation points, which is common in growing rod constructs. They noted that upper screws might be prone to implant failure in such spanning constructs that are not formally fused.

To the best of our knowledge, this is the first study describing the prevalence and risk factors of spinal canal encroachment after pull-out of a pedicle screw in the proximal foundation of a dual GR construct. According to our results, spinal canal encroachment was seen in 11 of the 21 patients with upper thoracic pedicle screw pull-out $(52 \%)$. Comparison of the groups revealed that coronal plane deformity at the time of screw pull-out had increased in all patients; however, this increase was significantly higher in patients with canal encroachment (45.7 vs. 35 degrees, $P=0.002$ ). In addition, while not statistically significant, PJA increased linearly in patients with canal encroachment but spiked at the time of pull-out in patients without canal encroachment $(P=0.269)$. 
The possible explanation for the medial migration of screws is the continued rotation of the vertebra on its own axis while the GR construct remained static. The convergent pedicle orientation at the upper thoracic spine and a possible unnoticed initial medial breach may have caused the medial migration of the screw after pull-out while traveling posteriorly. ${ }^{17}$ Progressive medial screw migration may have then occurred, with continued worsening of vertebral rotation caused by the loss of anchor purchase. Simultaneously with screw pull-out, the relative orientation of the screw to the vertebra was seen to be altered and pedicle screws were found to have migrated into the spinal canal. There was no neurological deficit, likely because of the slow progression of the phenomenon. The trend of changes in coronal deformity magnitude and PJA in our patients with and without canal encroachment supports this theory.

As a major drawback of this study, there is no CT confirmation of the position of the pedicle screws at the proximal foundation after index operation. At our institution, during freehand placement of pedicle screws, meticulous care is taken to verify all 4 walls of the intended screw track are intact. During initial instrumentation, this verification is performed by the senior surgeon, who probes the track with a ball-tipped instrument. In addition, neither fluoroscopy nor neurological monitoring raised suspicion for medial screw malposition and/or spinal cord compression during index surgery. However, as we do not routinely verify screw position in uncomplicated cases with CT scanning due to concerns for excessive radiation exposure, a medial breach at the time of insertion is impossible to be ruled out with absolute certainty. Other limitations of the study are its retrospective design and limited patient number that precludes a reliable statistical analysis to describe other risk factors.

In conclusion, pedicle screws may constitute a risk for spinal cord injury during follow-up in growing rods even though insertion is safe. A well-placed pedicle screw can migrate medially in case of pull-out. In patients with proximal anchor failure, especially if there is a notable increase of coronal deformity or a progression of PJA, possible spinal encroachment should be kept in mind. Preoperative CT evaluation and intraoperative neurological monitoring are highly recommended for revision surgery.

\section{REFERENCES}

1. Akbarnia BA, Breakwell LM, Marks DS, et al. Dual growing rod technique followed for three toeleven years until final fusion: the effect of frequency of lengthening. Spine (Phila Pa 1976). 2008; 33:984-990.

2. Akbarnia BA, Emans JB. Complications of growth-sparing surgery in early onset scoliosis. Spine (Phila Pa 1976). 2010;35:2193-2204.

3. Bess S, Akbarnia BA, Thompson GH, et al. Complications of growing-rod treatment for early-onset scoliosis: analysis of one hundred and forty patients. J Bone Joint Surg Am. 2010;92: 2533-2543.

4. Watanabe K, Uno K, Suzuki T, et al. Risk factors for complications associated with growing-rod surgery for early-onset scoliosis. Spine (Phila Pa 1976). 2013;38:464-468.

5. Watanabe K, Suzuki U, Kawakami N, et al. Risk factors for proximal junctional kyphosis associated with dual-rod growing-rod surgeryfor early-onset scoliosis. Clin Spine Surg. 2016;29:428-433.

6. Ranade A, Shamdani AF, Williams R, et al. Feasibility and accuracy of pedicle screws in children younger than eight years of age. Spine (Phila Pa1976). 2009;34:2907-2911.

7. Olgun ZD, Demirkiran G, Ayvaz M, et al. The effect of pedicle screw insertion at a young age on pedicle and canal development. Spine (Phila Pa 1976). 2012;37:1778-1784.

8. Ruf M, Harms J. Pedicle screws in 1- and 2-year-old children: technique, complications, and effect on further growth. Spine (Phila Pa1976). 2002;27:460-466.

9. Mahar AT, Bagheri R, Oka R, et al. Biomechanical comparison of different anchors (foundations) for the pediatric dual growing rod technique. Spine J. 2008;8:933-939.

10. Liljenqvist U, Hackenberg L, Link T, et al. Pull-out strength of pedicle screws versus pedicle and laminar hooks in the thoracic spine. Acta Orthop Belg. 2001;67:157-163.

11. Akbarnia BA, Yaszay B, Yazici M, et al. Biomechanical evaluation of 4 different foundation constructs commonly used in growing spine surgery: are rib anchors comparable to spine anchors? Spine Deform. 2014;2:437-443.

12. Myung KS, Skaggs DL, Johnston CE, et al. The use of pedicle screws in children 10 years of age and younger with growing rods. Spine Deform. 2014;2:471-474.

13. Alanay A, Cil A, Acaroglu E, et al. Late spinal cord compression caused by pulled-out thoracic pedicle screws: a case report. Spine (Phila Pa 1976). 2003;28:506-510.

14. Skaggs KF, Brasher AE, Johnston CE, et al. Upper thoracic pedicle screw loss of fixation causing spinal cord injury: a review of the literature and multicenter case series. J Pediatr Orthop. 2013; 33:75-79.

15. Kim YJ, Lenke LG, Bridwell KH, et al. Free hand pedicle screw placement in the thoracic spine: is it safe? Spine (Phila Pa 1976). 2004;29:333-342.

16. Hicks JM, Singla A, Shen FH, et al. Complications of pedicle screw fixation in scoliosis surgery: a systematic review. Spine (Phila Pa 1976). 2010;35:465-470.

17. Ebraheim NA, $\mathrm{Xu} \mathrm{R}$, Ahmad M, et al. Projection of the thoracic pedicle and its morphometric analysis. Spine (Phila Pa 1976). 1997;22:233-238 